\title{
A clinical syndrome of rostral and caudal spinal injury: neurological, neurophysiological and urodynamic evidence for occult sacral lesion
}

\author{
ALEKSANDAR BERIĆ, ${ }^{*} \dagger$ MILAN R DIMITRIJEVIĆ, ${ }^{*} \dagger$ J KEITH LIGHT $\ddagger$ \\ From the Department of Clinical Neurophysiology, ${ }^{*}$ The Institute for Rehabilitation and Research, and the \\ Department of Rehabilitation $\dagger$ and the Scott Department of Urology, $\ddagger$ Baylor College of Medicine, Houston, \\ Texas, USA
}

SUMMARY Patients with spinal cord injury show upper motor neuron dysfunction below the level of the lesion. Some patients with cervical and high thoracic injuries show unexpected lower leg atrophy and ankle jerk abnormalities together with persistence of urinary retention. Clinical, neurophysiological and urodynamic findings in 130 patients with cervical and thoracic injuries showed that 18 patients had additional lumbosacral dysfunction. Three patients had radiological findings demonstrating a second lesion of the lower spine. The remaining 15 patients, however, did not have any obvious bony lesion to account for the lumbosacral dysfunction. Atypical neurological findings, abnormal neurophysiological testing and aberrant detrusor behaviour were the essence of the occult lumbosacral dysfunction in cervical and thoracic spinal cord injury patients. Recognition of the presence of a double lesion was important for care of the neuropathic bladder and pain in addition to understanding the unexpected clinical signs.

Patients with chronic cervical and thoracic spinal cord injury typically show characteristics of upper motor neuron dysfunction below the level of the lesion. These include spontaneous muscle spasm, increased muscle tone, exaggerated tendon jerks, clonus, and detrusor hyperreflexia. ${ }^{12}$ There is usually no dysfunction of segmental reflexes on clinical or neurophysiological testing. The lumbosacral somatosensory evoked potentials (LSEPs) are retained ${ }^{3}$ while cortical SEPs show varying degrees of abnormality. ${ }^{4}$ Contrary to these findings, some patients with high spinal cord injury have altered or even absent LSEPs, as occurs in cauda equina injury. ${ }^{5}$ Careful clinical evaluation of these patients often reveals relatively diminished spasticity and moderate atrophy of the lower leg muscles together with the persistence of urinary retention two or more years post injury. ${ }^{6}$ These atypical clinical observations suggest the presence of a lesion at the sacral level of the

Address for reprint requests: A Berić MD, Department of Clinical Neurophysiology, The Institute of Rehabilitation and Research, 1333 Moursund Avenue, Houston, Texas 77030.

Received 8 July 1986 and in revised form 2 October 1986. Accepted 9 October 1986 spinal cord, in addition to the major rostral spinal injury. A systematic investigation of patients with cervical and thoracic spinal cord injury was performed for signs of a lumbosacral lesion.

\section{Material and methods}

One hundred and thirty traumatic chronic spinal cord injury patients were included in this prospective study. This represented the total number of patients seen consecutively for spasticity or pain evaluation and treatment from September 1983 to September 1985 with the neurological level of the lesion at or above T8. At the time of evaluation, the patients were in a stable condition without decubitus ulcers or infections and were at least 10 months after injury. Some were taking antispastic medication but the dosage had not been altered for at least 2 months prior to the evaluation.

A conventional neurological examination was performed, including a thorough interview about positive sensory symptoms, especially pain, its type and distribution. Neurophysiological evaluation consisted of recording lumbosacral somatosensory evoked potentials with the electrodes placed over the lumbosacral vertebrae (S-1, L-4, L-2, and T-12), with the reference electrode at T6. Recordings from T10 were also obtained when indicated. Two different intensities of stimulation of the tibial nerve at the knee were used; maximal $\mathrm{H}$ reflex level and maximal $\mathrm{M}$-wave of the soleus 
Table 1 Patients with radiologically confirmed double spinal injury

\begin{tabular}{|c|c|c|c|c|c|c|c|c|c|c|c|c|c|c|c|c|}
\hline & \multirow{3}{*}{$\begin{array}{l}\text { Age } \\
(y r)\end{array}$} & \multirow[b]{3}{*}{ Level } & \multirow{3}{*}{$\begin{array}{l}\text { Clin } \\
M / S^{*}\end{array}$} & \multirow[b]{3}{*}{ Post } & \multicolumn{4}{|c|}{ LSEPS } & \multirow{2}{*}{\multicolumn{2}{|c|}{ Ankle jerk }} & \multirow{2}{*}{\multicolumn{2}{|c|}{ Knee jerk }} & \multirow{2}{*}{\multicolumn{2}{|c|}{ Plantar reflex }} & \multirow[b]{3}{*}{$C M G_{\ddagger}^{\ddagger}$} & \multirow{3}{*}{$\begin{array}{l}\text { Pain } \\
\text { cauda } \\
\text { equina }\end{array}$} \\
\hline & & & & & \multicolumn{2}{|l|}{$R$} & \multicolumn{2}{|l|}{$L$} & & & & & & & & \\
\hline & & & & & $R \S$ & $S$ & $R \S$ & $S$ & $R$ & $L$ & $R$ & $L$ & $\bar{R}$ & $L$ & & \\
\hline 1. & 57 & T3 & $\mathrm{C} / \mathrm{C}$ & 10 & 0 & 0 & 0 & 0 & 0 & 0 & 0 & 0 & 0 & 0 & AREFL & No \\
\hline 2. & 20 & C3 & $\mathrm{I} / \mathrm{I}$ & 10 & $\mathrm{~N}$ & & 0 & 0 & $\mathrm{E}$ & 0 & $\mathrm{E}$ & $\mathrm{E}$ & + & 0 & UNSUS & No \\
\hline 3. & 50 & 17 & $\mathrm{C} / \mathrm{C}$ & 13 & 0 & 0 & 0 & 0 & 0 & 0 & 0 & $\mathbf{E}$ & 0 & 0 & AREFL & No \\
\hline
\end{tabular}

*Clinical assessment of motor (M) and sensory (S) dysfunction.

†Time after injury in months.

† See Material and methods for key.

$\S R$ component of LSEP.

muscle. Teca preamplifier amplifier system (PA63-AA6 MK3) was used with amplification of 50,000. Analog filters were set to $8 \mathrm{~Hz}-3200 \mathrm{~Hz}$. Analysis time was $40 \mathrm{~ms}$ and sampling interval $80 \mu \mathrm{s}$. One hundred and twenty eight responses were averaged on two separate occasions by a Hewlett Packard 1000 Computer System, which was also used for permanent storage and analysis. $\mathrm{R}$ and $\mathrm{S}$ waves of the LSEP were analysed separately for latency and amplitude. Knee and ankle jerks were elicited by handheld electromagnetic hammer and recorded with surface $\mathrm{EMG}^{7}$ of the quadriceps and triceps surae muscles. The plantar reflex was elicited by dragging a ball-point pen with a micro switch along the plantar surface ${ }^{8}$ and recorded with polyelectromyography of the lower extremities. Surface electrodes were placed bilaterally over tibialis anterior, triceps surae, quadriceps, hamstrings, adductors, abdominal and lumbar paraspinal muscles. Additional electromyography and recording of sensory nerve action potentials were obtained in patients in whom there was clinical suspicion of a lumbosacral lesion. Cortical somatosensory evoked potentials from tibial nerve stimulation were recorded in all the patients using a silver chloride cup electrode placed over $\mathrm{Cz}^{\prime}$ point (modified $\mathrm{Cz}$ ) referenced to $\mathrm{Fz}$ (International $10 / 20$ system). The $\mathrm{Cz}^{\prime}$ point was $1.5 \mathrm{~cm}$ behind $\mathrm{Cz}$. One hundred and twenty eight responses were averaged on two separate occasions with the analysis time of $160 \mathrm{~ms}$ and sampling interval of $312 \mu \mathrm{s}$. Twenty neurologically healthy subjects served as controls for recording of LSEPs and were age matched with the spinal cord injury patients.

Urodynamic evaluation was performed using pressureflow studies with simultaneous video-cystourethrography. The results were recorded on a DISA 2100 urodynamic instrument. The filling rate in all patients was $60 \mathrm{ml} / \mathrm{min}$. A slow filling rate of $10 \mathrm{ml} / \mathrm{min}$ was used in addition when indicated. An 8 French, double lumen Porges catheter was inserted transurethrally into the bladder. One channel was connected to a Statham transducer to monitor intravesical pressures while the other was attached to an infusion pump for constant bladder filling. A rectal catheter was inserted to monitor intra-abdominal pressures. Subtracted intravesical pressure was performed automatically on the DISA 2100 . A concentric needle electrode was inserted percutaneously to

Table 2 Patients with neurophysiological signs of lumbosacral abnormality

\begin{tabular}{|c|c|c|c|c|c|c|c|c|c|c|c|c|c|c|c|c|}
\hline & \multirow{3}{*}{$\begin{array}{c}\text { Age } \\
(y r)\end{array}$} & \multirow[b]{3}{*}{ Level } & \multirow{3}{*}{$\begin{array}{l}\text { Clin* } \\
M / S\end{array}$} & \multirow[b]{3}{*}{ Post } & \multicolumn{4}{|c|}{$L S E P s$} & \multirow{2}{*}{\multicolumn{2}{|c|}{ Ankle jerk }} & \multirow{2}{*}{\multicolumn{2}{|c|}{ Knee jerk }} & \multirow{2}{*}{\multicolumn{2}{|c|}{ Plantar reflex }} & \multirow[b]{3}{*}{$C M G_{+}^{+}$} & \multirow{3}{*}{$\begin{array}{l}\text { Pain } \\
\text { cauda } \\
\text { equin }\end{array}$} \\
\hline & & & & & \multicolumn{2}{|l|}{$R$} & \multicolumn{2}{|l|}{$L$} & & & & & & & & \\
\hline & & & & & $R \S$ & $S$ & $R \S$ & $S$ & $\boldsymbol{R}$ & $L$ & $R$ & $L$ & $R$ & $L$ & & \\
\hline \multicolumn{17}{|c|}{ (A) } \\
\hline 1. & 28 & C4 & $\mathrm{C} / \mathrm{C}$ & 65 & 0 & 0 & 0 & $\downarrow \downarrow$ & 0 & 0 & $E$ & $\mathbf{E}$ & 0 & 0 & HYPO & Yes \\
\hline 2. & 34 & T6 & $\mathrm{C} / \mathrm{C}$ & 33 & 0 & 0 & 0 & 0 & 0 & 0 & $\mathbf{E}$ & $\overrightarrow{\mathbf{E}}$ & + & + & UNSUS & No \\
\hline 3. & 45 & $\mathrm{~T} 4$ & $\mathbf{C} / \mathbf{C}$ & 11 & 0 & $\downarrow$ & 0 & 0 & 0 & $\downarrow$ & $\overline{\mathbf{E}}$ & $\overrightarrow{\mathbf{E}}$ & 0 & + & HYPER & Yes \\
\hline 4. & 27 & T5 & $\mathrm{C} / \mathrm{C}$ & 60 & 0 & 0 & 0 & 0 & $\downarrow$ & 0 & $\mathrm{E}$ & $\mathbf{E}$ & +- & +- & HYPER & No \\
\hline 5. & 34 & C4 & $\mathrm{I} / \mathrm{I}$ & 122 & 0 & $\downarrow$ & 0 & 0 & $\downarrow$ & 0 & E & $\downarrow$ & + & + & AREFL & Yes \\
\hline 6. & 19 & T8 & $\mathrm{C} / \mathrm{C}$ & 15 & 0 & 0 & 0 & 0 & $\downarrow$ & 0 & $\mathrm{E}$ & $\mathbf{E}$ & + & + & AREFL & No \\
\hline 7. & 40 & C6 & $\mathrm{I} / \mathrm{I}$ & 58 & 0 & 0 & 0 & 0 & 0 & $\downarrow$ & $\downarrow$ & $\mathbf{E}$ & 0 & 0 & UNSUST & No \\
\hline 8. & 21 & C6 & $\mathrm{C} / \mathrm{C}$ & 17 & $\downarrow$ & $\downarrow$ & $\mathbf{N}$ & $\mathbf{N}$ & 0 & 0 & E & $\overline{\mathbf{E}}$ & 0 & +- & HYPO & Yes \\
\hline 9. & 43 & $\mathrm{~T} 4$ & $\mathrm{C} / \mathrm{C}$ & 63 & & $\downarrow$ & $\downarrow$ & $\downarrow$ & E & $E$ & $\downarrow$ & $\downarrow$ & + & + & HYPO & No \\
\hline 10. & 32 & C7 & $\mathrm{C} / \mathrm{I}$ & 182 & $\downarrow \downarrow$ & $\downarrow$ & $\mathbf{N}$ & $\mathbf{N}$ & 0 & 0 & E & $\mathbf{E}$ & + & + & No test & Yes \\
\hline 11. & 24 & C5 & $\mathrm{C} / \mathrm{C}$ & 23 & 0 & $\rightarrow$ & 0 & $\vec{a}$ & 0 & 0 & 0 & 0 & 0 & +- & HYPO & Yes \\
\hline 12. & 20 & $\mathrm{~T} 1$ & $\mathrm{C} / \mathrm{I}$ & 14 & $\downarrow$ & $\downarrow$ & 0 & 0 & 0 & 0 & $\mathbf{E}$ & $\mathbf{E}$ & + & + & AREFL & No \\
\hline \multicolumn{17}{|l|}{ (B) } \\
\hline 13. & 20 & $\mathrm{~T} 2$ & $\mathrm{C} / \mathrm{C}$ & 14 & $\mathbf{N}$ & D & $\mathbf{N}$ & D & E & $E$ & E & $\mathbf{E}$ & + & + & AREFL & Yes \\
\hline 14. & 28 & C5 & $\mathrm{C} / \mathrm{I}$ & 10 & $\mathbf{N}$ & $\downarrow$ & $\mathbf{N}$ & $\downarrow$ & $\downarrow$ & $\downarrow$ & $\mathbf{E}$ & $\mathbf{E}$ & + & + & AREFL & No \\
\hline 15. & 23 & C6 & $\mathrm{I} / \mathrm{C}$ & 21 & $\mathbf{N}$ & D & $\mathbf{N}$ & D & E & E & $\mathbf{E}$ & $\mathbf{E}$ & + & + & HYPO & No \\
\hline
\end{tabular}

*Clinical assessment of motor (M) and sensory (S) dysfunction.

†Time after injury in months.

+See Material and methods for key.

$\S \mathrm{R}$ component of LSEP. 

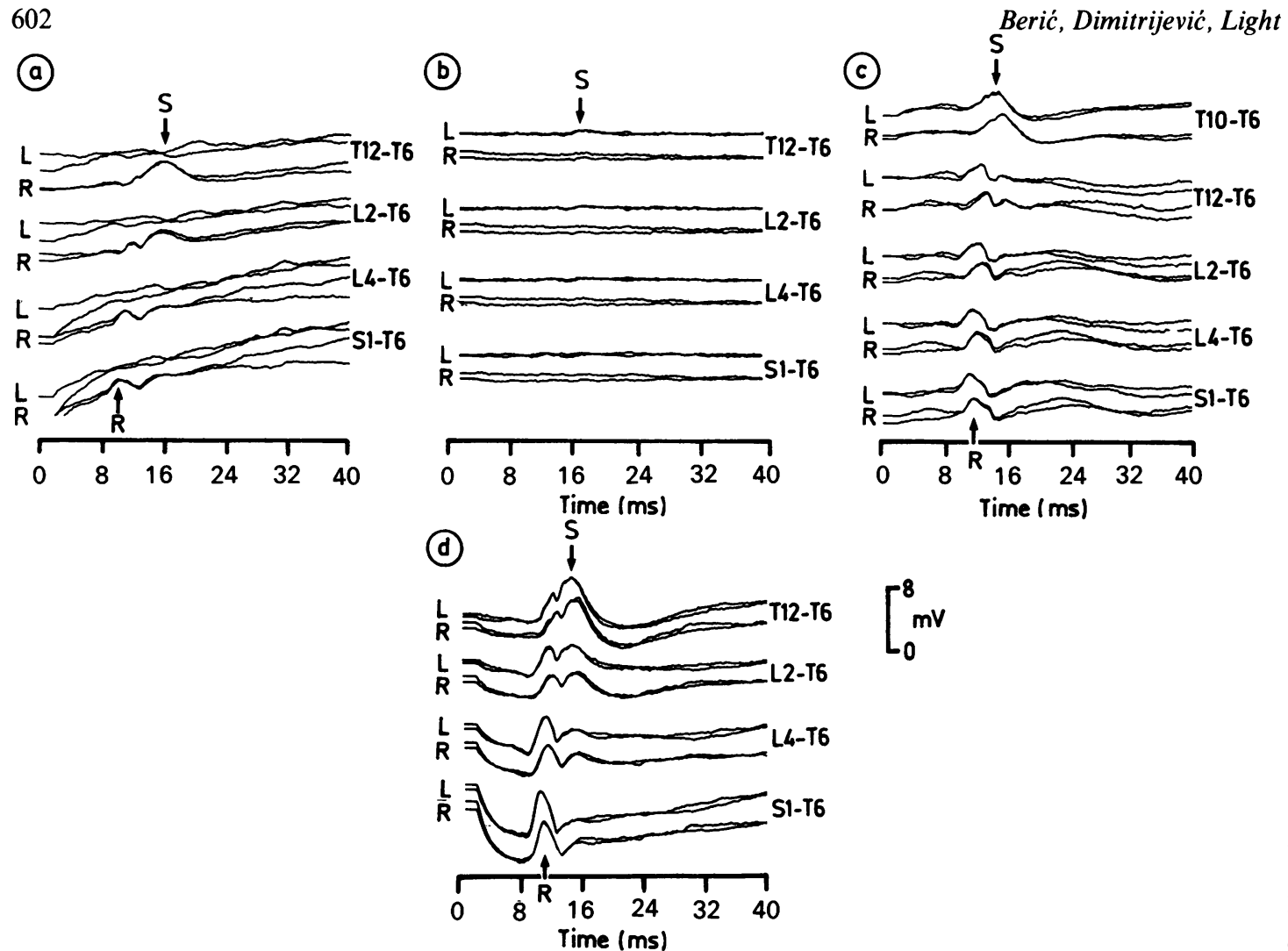

Fig 1 (a) LSEPs of a patient with left sacroiliac fracture. Note also a dispersed $S$ wave from the "healthy" right side.

(b) Absent LSEPs in patient with severe lumbosacral dysfunction without lower spine fracture. Note a trace of $S$ wave on the left side. (c) LSEPs from patient with delayed return of bladder contractility with abnormally high vertebral position of $S$ wave (T10). (d) Normal LSEPs from a patient with a C6 spinal cord injury and no signs of lumbosacral dysfunction. The intensity of electrical stimulation in $A, B, C$ and $D$ was adjusted for maximal $M$ response of the soleus muscle.

monitor activity of the periurethral striated muscle. Videocystourethrography was obtained in the left lateral position at pertinent times during the study to assess opening of the bladder neck and anatomy of the posterior urethra. All patients who had not developed spontaneous voiding or adequate bladder emptying underwent urodynamic assessment 6 months after injury and subsequently at yearly intervals. This evaluation was repeated in patients with acontractile and hypocontractile bladders up to 30 months after injury. Neurophysiological testing was performed as shown in tables 1 and 2, and approximately at the same time urodynamic testing was repeated.

Criteria for abnormalities of the observed parameters were as follows: the latencies of the $R$ and $S$ components of the LSEPs were considered abnormal if greater than 2.5 SD from the mean of the control population $(\rightarrow)$. The amplitude was considered abnormally small if greater than 2 SD below the control values $(\downarrow)$. A trace response was labelled as extremely diminished with two down arrows. Absent responses were labelled 0 . If the $S$ wave was found two or more vertebral levels more proximal than in a normal population, it was considered abnormally displaced (D). Ankle and patellar jerks were analysed from the amplitudes of the surface EMG responses in addition to the clinical assessment. A response above $300 \mu \mathrm{V}$ was labelled exaggerated (E), below $100 \mu \mathrm{V}$ diminished ( $\downarrow$ ), and absent as 0 . The plantar reflex was analysed clinically and neurophysiologically for the distribution toward proximal muscle leg groups and for amplitude of the components. Withdrawal, with activity in the proximal and distal muscles associated with a clinically observed hip flexion was labelled as present $(+)$. Activity in the distal leg muscles alone with an upgoing hallux or toe flexion clinically was considered diminished $(+-)$, and absent with no EMG activity and no clinical response $(0)$. The threshold of the detrusor contraction together with the maximum intravesical pressure (Piso) were calculated from the pressure-flow studies. Detrusor hypocontractility was defined as a Piso of $45 \mathrm{~cm}$ water or less as measured from the total bladder pressure (HYPO). The detrusor contraction was considered as abnormally short with a duration of less than $15 \mathrm{~s}$ (UNSUS). The presence of a pain, compatible with a cauda equina distribution was considered as YES, and no pain in the lumbosacral region as NO. It should be stressed that the pain distribution was totally separate from any more proximal abdominal or transitional zone pain. 
Results

From 130 patients studied, 18 had abnormal or absent LSEPs suggesting lumbosacral dysfunction in addition to their cervical or thoracic injury (tables 1 and 2). Three patients with obvious signs of a lumbosacral lesion are presented in table 1 for comparison. The first patient suffered multiple bullet injuries, one of which lodged in the sacral canal. The other two, who were injured in motor vehicle accidents, had radiological fractures involving the lumbosacral and sacroiliac areas respectively. All three had profoundly

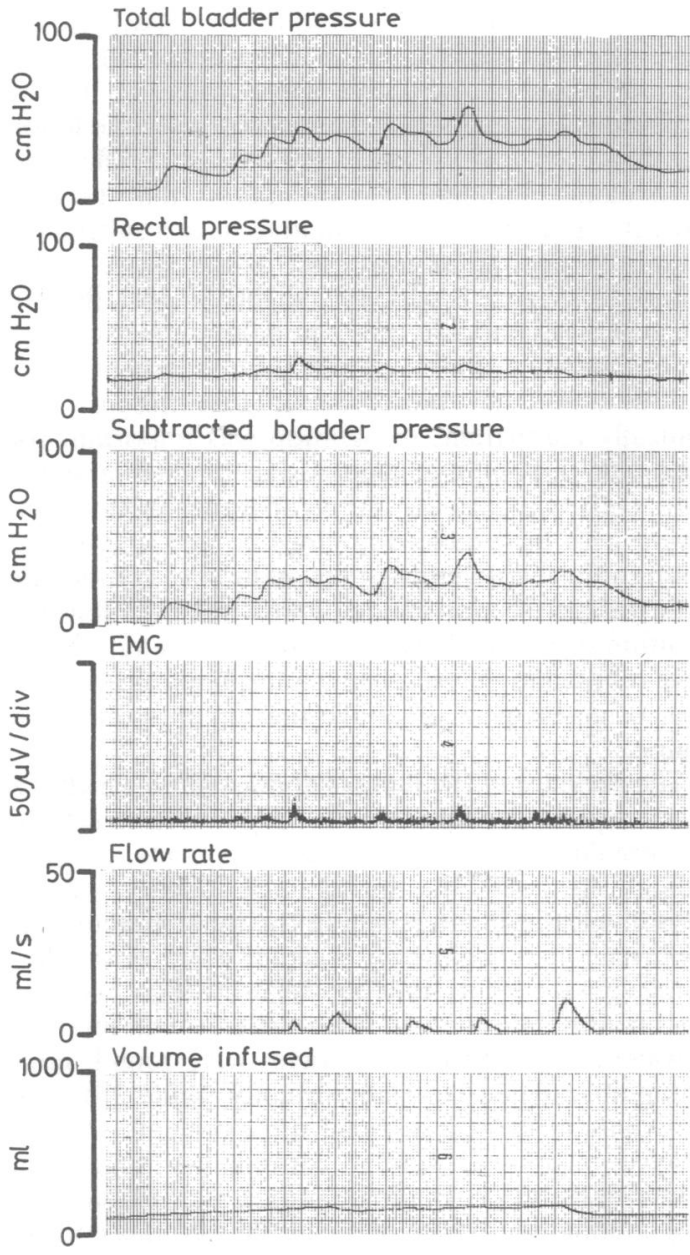

Fig 2 Bladder pressure-flow study in C6 spinal cord injury patient with hypocontractile detrusor. Note low threshold of detrusor contraction $(120 \mathrm{ml})$, minimal external sphincted dyssynergia and presence of interrupted flow rate.
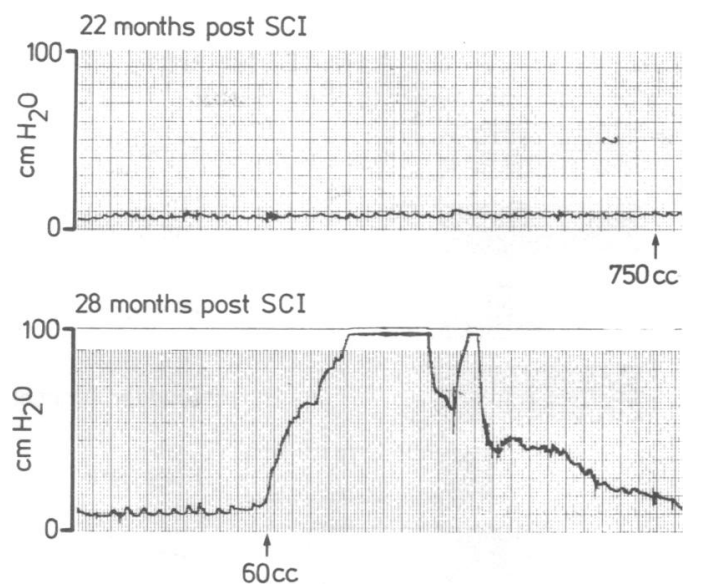

Fig 3 Cystometrogram of a T2 spinal cord injury patient with delayed return of a bladder contractility. Note detrusor areflexia at 22 months after injury, but a low threshold, high pressure, sustained contraction at 28 months after injury.

abnormal LSEPs and other signs of a lumbosacral lesion. The results of the LSEP study in patient 2 are shown in fig la.

Of the remaining 15 patients (table 2) with abnormal LSEPs, all had other signs of lumbosacral dysfunction. None of these 15 patients had a history of a second injury to the lumbosacral region or radiological findings of such a lesion. Five patients sustained their injury in a diving accident (patients 8, $11,12,14,15)$ while two patients were gunshot wounds (patients 1,13 ). The remainder were injured in motor vehicle accidents.

An abnormal $\mathrm{S}$ wave was present in all 15 patients. All but three showed an abnormality of the $R$ wave at the $S 1$ level indicating involvement of the root potential (table 2A). The LSEPs of patient 1 from table 2A are shown in fig $1 \mathrm{~b}$. Four patients (table $2 \mathrm{~A}$ ) had normal sural SNAPs (patients 1, 8, 11 and 12), while in one this was absent unilaterally (patient 5). The results of three patients without a $\mathbf{R}$ wave abnormality are shown in table $2 \mathrm{~b}$. Patients 1 and 3 had unusual cranial-caudal distribution of the $S$ wave, this being present over the surface electrode attached at the 10th thoracic vertebra spinous process and not at the usual 12th thoracic position (fig 1c). Patient 2 had normal distribution but diminished amplitude of the S waves.

The urodynamic pattern, done at six month intervals remained the same up to the time of the final neurophysiological and urodynamic profile (patients 5 and 10 did not have follow ups). The typical hypocontractile bladder response is shown in fig 2 (patient 8 ). Some patients, however ( 12 from table $2 a$ and 13 


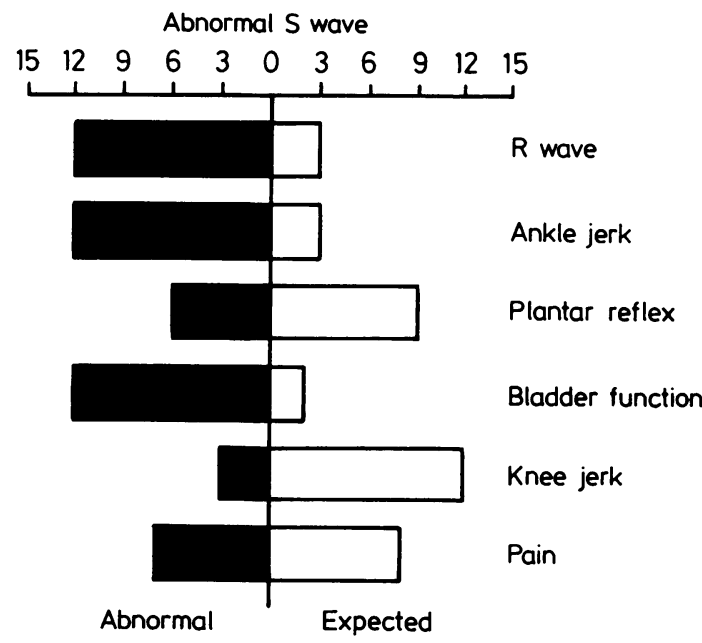

Fig 4 Relationship between abnormality of $S$ wave (LSEP) and clinical, neurophysiological and urodynamic findings in patients with lumbosacral dysfunction and absence of lower spine fracture. The right side shows the expected upper motor neuron dysfunction in high spinal cord injuries. On the left (shaded bars) are patients demonstrating atypical neurological, abnormal neurophysiological and aberrant detrusor behaviour considered abnormal for high spinal cord injuries.

and 14 from table $2 b$ ), had unusual urodynamic findings. Detrusor areflexia was present at the initial urodynamic evaluation 6 months after injury and at the time or after the neurophysiological profile (table 2). Repeat urodynamic follow up at 15, 28 and 19 months after injury showed a typical sustained bladder contraction with high pressures and a low threshold, findings characteristic of detrusor hyperreflexia associated with a supra-sacral lesion. Urodynamic follow up of patient 13 is shown in fig 3 . In addition, patient 15 (table $2 \mathrm{~b}$ ) who had detrusor hypocontractility up to 21 months after injury, developed typical hyperreflexia 28 months after injury.

The $\mathrm{S}$ wave abnormality was a constant finding in the patients with additional sacral dysfunction (table 2). The relationship with other observed parameters is summarised in fig 4 . The $R$ wave and ankle jerk abnormality showed the highest correlation with the abnormality of the $\mathrm{S}$ waves, while the plantar reflex was variable. In contrast, the majority of knee jerks were present. Bladder dysfunction also correlated well but there were two exceptions with typical UMN detrusor hyperreflexia (patients 3 and 4).

\section{Discussion}

Upper motor neuron dysfunction below the spinal cord lesion is characterised by disinhibition and/or excessive facilitation of spinal reflex activity. This also applies to patients with partial or complete absence of both voluntary and postural control. Thus, a mild or moderate lesion involving the spinal segmental reflex arc, with consequent diminished spinal cord hyperactivity, would be clinically unrecognised unless there were diagnostic techniques available for assessment of afferent input and spinal cord response below the level of the lesion. In our study, 18 of 130 patients with high thoracic and cervical cord injuries had abnormal lumbosacral somatosensory evoked potentials together with some clinical, neurophysiological, or urodynamic abnormality in various combinations. Each patient showed an abnormality in at least two independent objective tests, indicating lumbosacral radiculopathy and/or myelopathy. Only three cases had radiological findings demonstrating a second lesion of the lower spine (table 1). In the remaining 15 patients, the diagnosis of a second lesion was based on LSEPs, ankle jerks, plantar reflex and urodynamic findings. It is of interest that $14 \%$ of patients in this series were found to have a subclinical sacral dysfunction as the result of the above evaluation. This figure is probably slightly higher than in the general spinal cord injury population because our series consists of more severely injured patients.

Although the lumbosacral dysfunction was clinically inapparent, recognition was important for adequate care of the neuropathic bladder and the pain. Only two patients had bladder dysfunction consistent with the expected findings in high spinal cord injuries. In eight patients the pain was compatible with the type seen in cauda equina injury. ${ }^{9}$ Non recognition of the lumbosacral dysfunction could easily have resulted in the pain being interpreted as a central pain originating at or above the upper spinal cord injury.

The presence of the $\mathbf{R}$ wave abnormality, which is a cauda equina root potential, ${ }^{10}$ suggests a structural abnormality. This was seen in 15 patients. As it is difficult to assess the spinal cord function neurophysiologically in cases where the input potential is abnormal, it is unknown whether there was additional spinal cord dysfunction separate from the root dysfunction. However, in three patients with retained $\mathrm{R}$ waves, only abnormal $\mathrm{S}$ waves were found, indicating an isolated cord abnormality (table $2 b$ ). The urodynamic findings in these three patients showed delayed return of bladder contractility suggesting milder dysfunction, in contrast to those with signs of root dysfunction where only one showed this course. The remaining patients with an $\mathbf{R}$ wave abnormality never recovered their detrusor reflex. This is in agreement with the previous report describing delayed return of bladder function (average of 16.6 months 
post injury) provided there was no definite abnormality found with pelvic floor EMG. ${ }^{6}$

The above findings certainly suggest a double spinal dysfunction. It consists of atypical neurological findings, abnormal neurophysiological testing and aberrant detrusor behaviour. The severity of the dysfunction varied, but was always associated with abnormal LSEPs and ankle jerk findings. It is emphasised that the dysfunction was not in continuity with a more proximal injury, because of the presence of knee jerks and proximal leg spasticity. This supports the concept that the lumbosacral abnormality is a separate entity from the higher, primary spinal cord injury.

The pathomorphological basis of the sacral dysfunction is not yet fully understood. In the absence of specific evidence, one assumption is a traumatic lesion, but initial decreased spinal blood flow with consequent nerve hypoxia is also a possibility. None of the patients showed a clinical picture compatible with peripheral neuropathy or non-traumatic root compression. Syringomyelia is highly unlikely in our patients as there was no change in neurological function at or above the level of proximal lesion. In addition there was a clear separation of at least 10 to 20 spinal segments from the proximal level of the lesion. Furthermore, the majority of abnormal findings were compatible with cauda equina dysfunction below the spinal cord itself, thus excluding spinal cord pathology, such as syringomyelia being a major factor. Spinal stenosis could not be excluded, since myelography or CT were not performed, but is very unlikely in this group of young patients with normal radiological findings of the lower spine. Exploratory lumbar laminectomy was not undertaken in any case. Necropsy of the spinal cord injury patient with cervical and high thoracic lesion rarely includes examination in the cauda equina region. Kakulas (personal communication), described three cases of cervical spinal cord injury with signs of arachnoiditis in the sacral canal. It may be speculated that some initial direct or indirect injury to the cauda equina, without bony changes, triggered the arachnoiditis. Another possibility is that the blood from the primary injury, through deposition of haemosiderin in the superficial neural tissue triggered a toxic arachnoiditis. This was suggested as a cause of arachnoiditis by Gomori et al $^{11}$ in their report describing repeated haemorrhage from an ependymoma. Another relevant observation was made by Ragnarsson et al. ${ }^{12}$ They reported that one spinal cord injury patient, with probable tethering of the cord after a mid-thoracic injury, developed delayed myelopathy symptoms at some distance from the primary lesion. The symptoms diminished after cord section, allowing retraction of both ends. This surgical observation supports the previous necropsy finding by Bedbrook ${ }^{13}$ who described tethering of the cord between two fixed points in spinal cord injury. This is an interesting hypothesis as two patients in our series (patients 13 and 15) had findings on LSEP suggesting an abnormally high position of the cord. Further work is necessary, however, to confirm this and exclude the association with a tethered cord. In addition, further radiological examinations (MRI and CT) are necessary to clarify this hypothesis.

\section{Addendum}

After the study was completed we had an opportunity to evaluate two additional patients with cauda equina dysfunction as in group $2 \mathrm{~A}$ (table 2) radiologically. In the first patient metrizimide myelography showed irregularity of the sacral sac suggesting arachnoiditis. The other patient had an MRI study which revealed a central bulge of the L4-5 annulus with a decrease in both the disc space and sagittal diameter of the spinal canal. These two cases with a proven radiological abnormality may be representative of the double spinal dysfunction. However, it is possible that there is more than one cause for the sacral dysfunction in cervical and high thoracic SCI.

We thank Drs Carter, Donovan, Halstead and Meier from the Institute for Rehabilitation and Research Houston, Texax for referring patients for evaluation. Special thanks are due to $\mathrm{Dr} U$ Lindblom, Department of Neurology, Karolinska Sjukhuset, Stockholm, for critical review of the manuscript; Mrs Helen Spencer and Miss Debra Bernhard are thanked for editorial assistance. The support for this work was provided by the V.L. Smith Foundation for Restorative Neurology, Houston, Texas, the Rehabilitation Research and Training Center No 4 (Rehabilitation Services Administration Grant 16-P-56813-6) and Rehabilitation Services Administration Grant 13-P-59275-6.

\section{References}

1 Adams RD, Victor M. Motor paralysis. In: Principles of Neurology. New York: McGraw Hill, 1981:33-41.

2 Guttman L. Patterns of reflex disturbances. In: Spinal Cord Injuries. Oxford: Blackwell Scientific Publications, 1976:241-59.

3 Lehmkuhl D, Dimitrijević MR, Renouf F. Electrophysiological characteristics of lumbosacral evoked potentials in patients with established spinal cord injury. Electroencephalogr Clin Neurophysiol 1984;59: 142-55.

4 Dimitrijević MR, Prevec TS, Sherwood AM. Somatosensory perception and cortical evoked potentials in established paraplegia. J Neurol Sci 1983;60:253-65.

5 Ertekin C, Mutlu R, Sarica Y, Uckardesler L. Electro- 
physiological evaluation of the afferent spinal roots and nerves in patients with conus medullaris and cauda equina lesions. J Neurol Sci 1980;48:419-33.

6 Light JK, Faganel J, Beric A. Detrusor areflexia in suprasacral spinal cord injuries. J Urol 1985;134: 295-7.

7 Dimitrijevic MR, Nathan PW. Studies of spasticity in man. 6. Habituation, dishabituation and sensitization of tendon reflexes in spinal man. Brain 1973; 96:337-54.

8 Dimitrijević MR, Nathan PW. Studies of spasticity in man. 1. Some features of spasticity. Brain 1967; 90:1-30.

9 White JC, Sweet WH. Pain of spinal origin. In: Pain and the Neurosurgeon. Springfield: Charles C Thomas
Publisher, 1969:435-47.

10 Dimitrijević MR, Larsson LE, Lehmkuhl D, Sherwood AM. Evoked spinal cord and nerve root potentials in humans using a non-invasive recording technique. Electroencephalogr Clin Neurophysiol 1978;45:331-40.

11 Gomori A, Grossman RI, Bilaniuk LT, Zimmerman A, Goldberg HI. High-field MR imaging of superficial siderosis of the central nervous system. $J$ Comput Assist Tomogr 1985;9:972-5.

12 Ragnarsson TS, Durward QJ, Nordgren RE. Spinal cord tethering after traumatic paraplegia with late neurological deterioration. J Neurosurg 1986;64:397-401.

13 Bedbrook G. Discussion in Proceedings of the 1970 Annual Scientific meeting of the International Medical Society of Paraplegia. Paraplegia 1971;9:35. 